\title{
EFFECTS OF CARBON MONOXIDE ON DPG CONCENTRATIONS IN THE ERYTHROCYTE
}

\author{
B. D. Dinman, M.D. \\ Institute of Environmental and Industrial Health \\ and \\ J. W. Eaton, Ph.D. and G. J. Brewer, M.D. \\ Department of Human Genetics, School of Medicine, The University of Michigan \\ Ann Arbor, Mich.
}

For many years 2,3-diphosphoglycerate (DPG) has been known to be generated in the erythrocyte by a shunt in the Embden-Myerhof pathway. Levels of DPG are very high in the eythrocyte compared to its concentration in other tissues. ${ }^{1}$ Because its function was unknown, DPG could have been characterized as "a substance in search of a function." Conversely, the greatly decreased oxygen affinity of intact red cells as compared to purified hemoglobin was "a function in search of an explanation." Now it appears that the "substance" and the "function" have come together. The pioneering work of Chanutin and Curnish ${ }^{2}$ and of Benesch and coworkers ${ }^{3}$ has shown that DPG complexes with reduced hemoglobin. This binding of DPG markedly decreases the oxygen affinity of the hemoglobin as compared with that of purified hemoglobin.

Similarly, it has been noted that the oxyhemoglobin dissociation curve is shifted toward the right in various types of hypoxia such as anemia and chronic pulmonary disease, ${ }^{4-6}$ and altitude. ${ }^{7}$ It has been subsequently demonstrated that this right shift is associated with marked elevations in red cell DPG concentrations. ${ }^{8}$

In view of the leftward (Haldane) shift of the oxyhemoglobin curve induced by $\mathrm{CO}$, possible interactions of the right shift associated with DPG alterations constituted an appropriate subject for inquiry. This is in keeping with the best tradition associated with $\mathrm{CO}$ research, i.e., the manipulation of this toxic gas in order to gain some insights into the inner workings of that remarkable tetrapyrrole, hemoglobin.

\section{Methods}

Twelve human volunteers were given doses of $\mathrm{CO}$ calculated to produce 15 to $25 \% \mathrm{COHb}$. Approximately three hours after the initial dose of $\mathrm{CO}, \mathrm{COHb}$ levels were estimated by the breathholding method of Jones and colleagues. ${ }^{9}$ with breath $\mathrm{CO}$ concentrations determined by the use of a nondispersive, Luft-type infra-red spectrophotometer (Lira Model 200, MSA), which utilizes a 1-meter cell. The correlation of alveolar $\mathrm{CO}$ with $\mathrm{COHb}$, the latter determined by the method of Collison and coworkers, ${ }^{10}$ appeared to follow the regression developed by Jones and coworkers. ${ }^{9}$

Prior to exposure to $\mathrm{CO}$, red cell DPG was determined by use of the chromatropic acid method of Bartlett ${ }^{11}$ as modified in our laboratories. ${ }^{12}$ This determination was repeated six hours after the first dose of $\mathrm{CO}$. Control determinations of DPG were made on samples obtained at the same times of day as the previous day's exposure procedure, i.e., 9 a.m. and 3 p.m.

In addition, 42 rats were exposed to approximately $0.8 \% \mathrm{CO}$ for ten to 15 minutes. This was sufficient to produce peak concentrations of $50-60 \% \mathrm{COHb}$, as estimated by the IL Co-oximeter. Twenty-two of this group of animals were 
Dinman et al.: DPG Concentrations

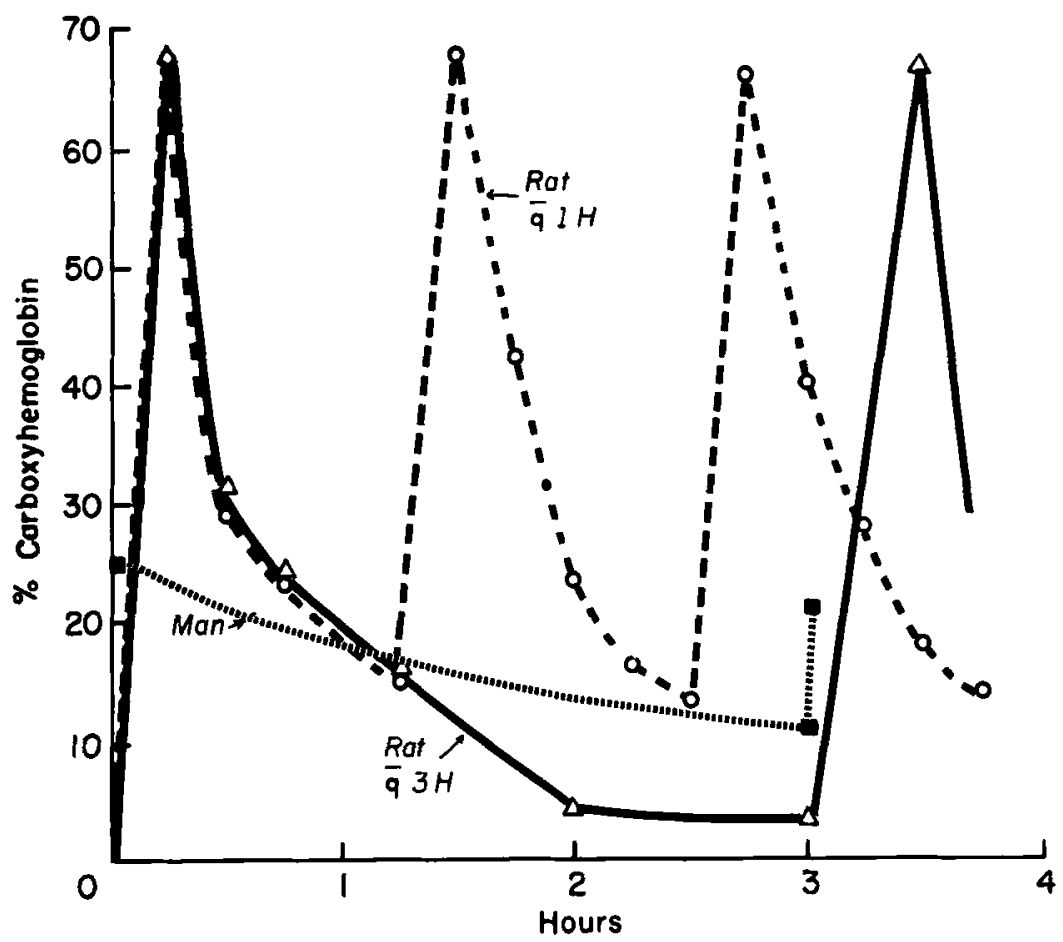

Figure 1. Dose regimens and mean COHb levels in rats and in man.

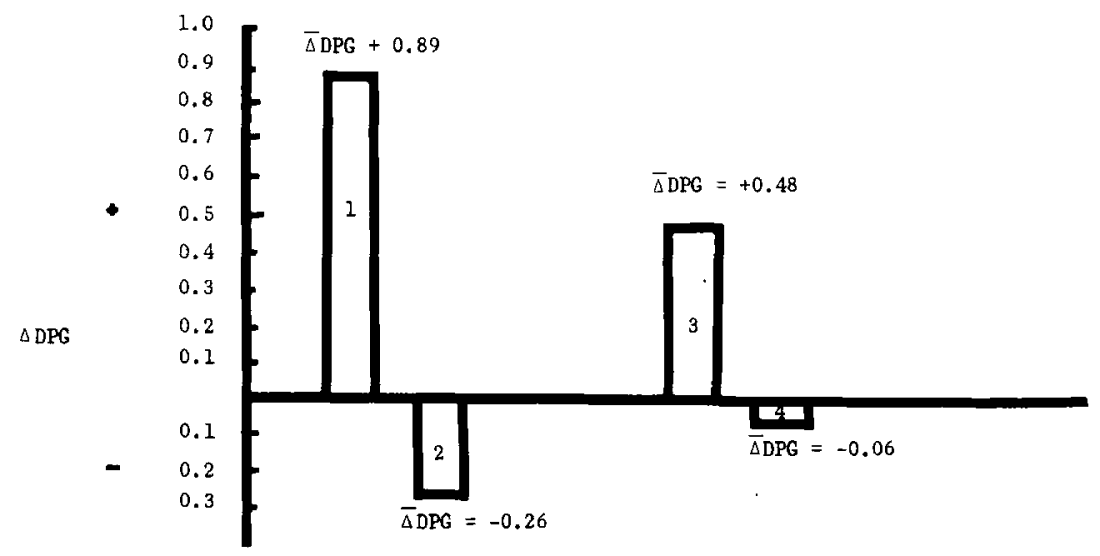

Subjects $1-12$

$$
\mathrm{n}=12
$$

1 Differs from 2

$=p<0.05$
Subjects $1-16$

$$
\mathrm{n}=16
$$

1 Differs from 2

$=\mathrm{N} . \mathrm{S}$.

Figure 2. Mean $\triangle$ DPG following CO exposure of men (Bar 1, 3) and during control periods (Bar 2, 4). 
TABLE 1

DPG Concentrations in Control and CO-Exposed Rats

\begin{tabular}{llcccc}
\hline Rats & $\mathrm{N}$ & $\begin{array}{c}\text { DPG Levels } \\
\text { Baseline }\end{array}$ & $6 \mathrm{hrs}$ & $\begin{array}{c}\text { Difference } \\
\text { (Mean } \pm \text { I.S.D.) }\end{array}$ & $\begin{array}{c}\text { t-Test on } \\
\text { Differences }\end{array}$ \\
\hline CO-treated & 22 & 21.3 & 23.5 & $+2.2( \pm 2.6)$ & $\mathrm{t}+3.49$ \\
Controls & 24 & 23.0 & 22.5 & $-0.5( \pm 2.7)$ & $\mathrm{p}<0.01$ \\
\hline
\end{tabular}

dosed every hour for five hours; another group was dosed only twice, i.e., at zero time and at three hours. The CO-treated rats and an equal number of control rats were sampled for DPG at the beginning of, and at the end of, the six th hour of the experiment. FIGURE 1 demonstrates the two different regimens and the mean levels of $\mathrm{COHb}$ achieved among the experimental animals and men.

\section{Results}

Analysis by student's t-test indicated that the level of DPG was elevated signi-

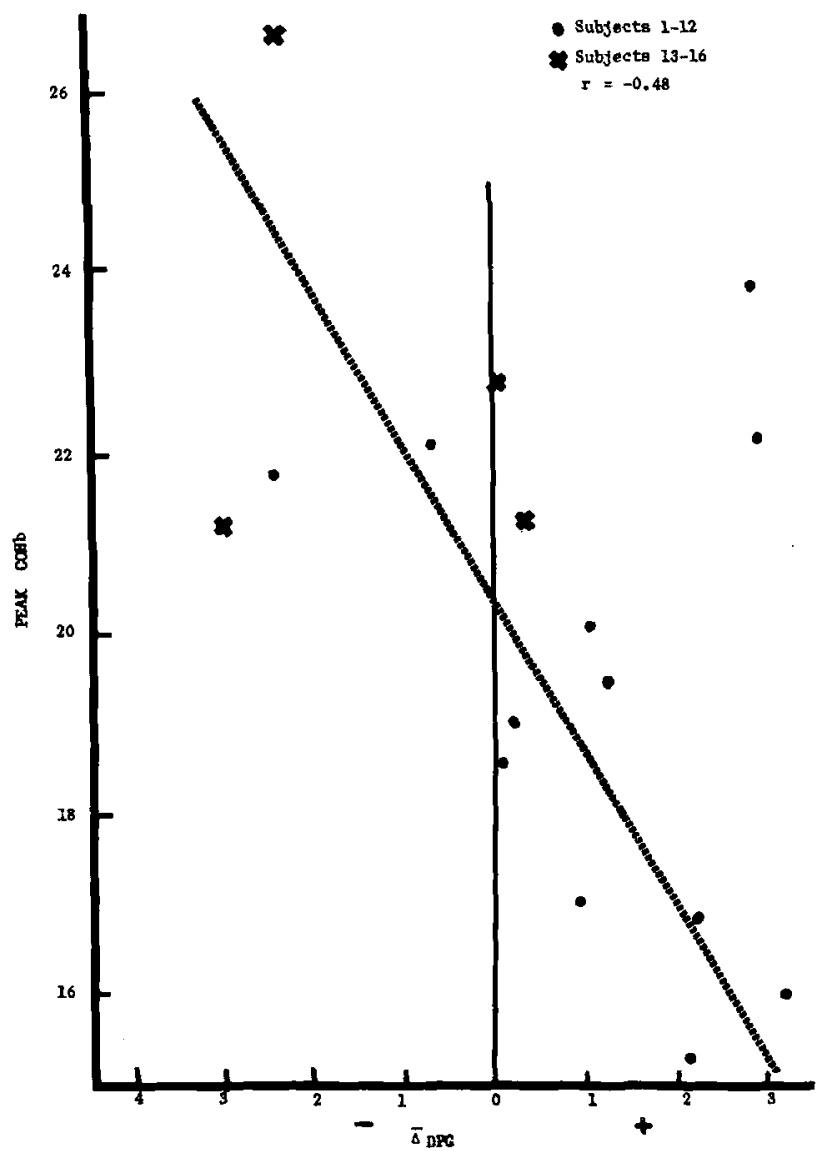

Figure 3. Peak COHb and $\triangle$ DPG among 16 men exposed to $C O$. 

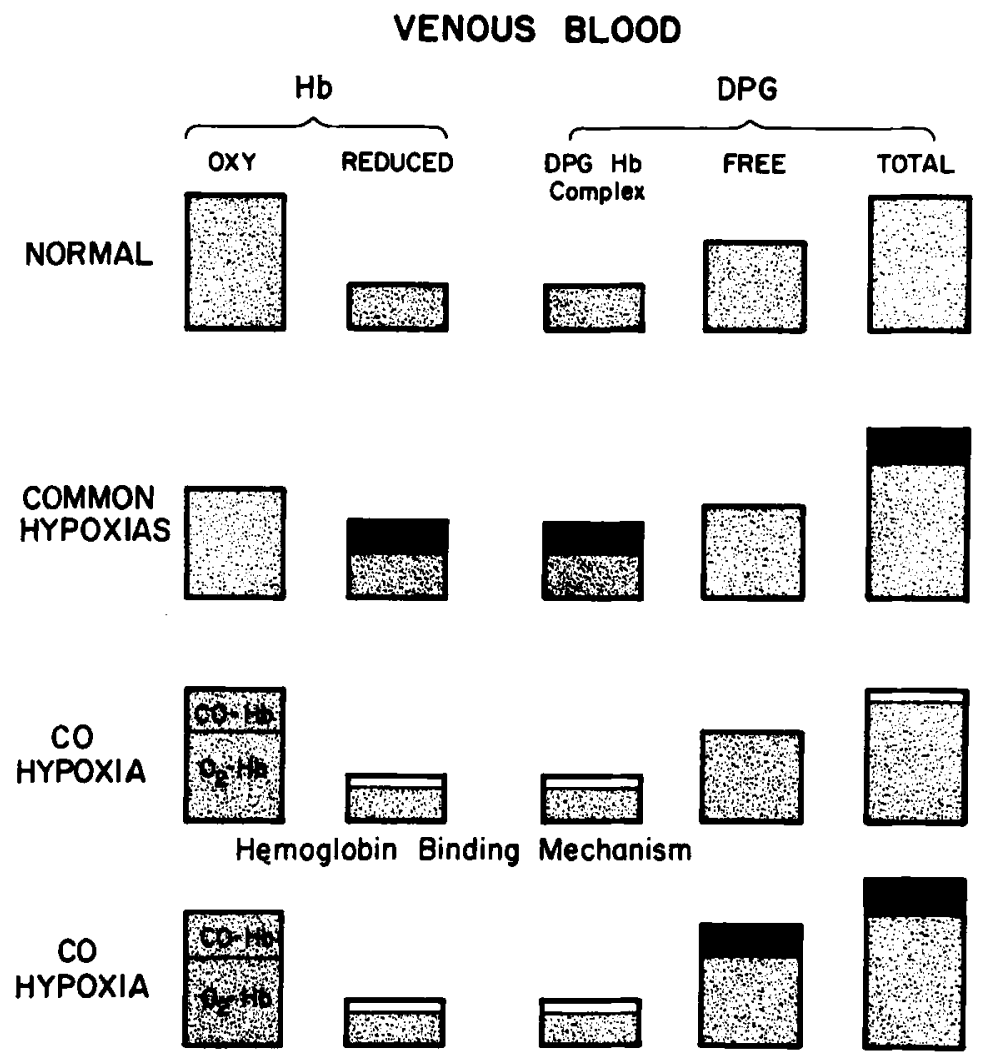

Hypoxia Mechanism

FIGURE 4. Distribution of free and total DPG in the erythrocyte in normal, common hypoxias, and $\mathrm{CO}$ loadings.

ficantly in CO-treated rats as compared with controls. This finding applied only to the rats given the two doses of $\mathrm{CO}$ three hours apart, rather than those exposed at the six hourly intervals (TABLE 1). (A preliminary report has been published..$^{13}$ )

Among the 12 human volunteers we found that while the control levels of DPG were constant or even tended to fall in the course of the day, upon exposure to CO there was a significant rise in DPG (FIGURE 2), as compared to change in DPG on nonexposure days. However, after exposing four more subjects to somewhat larger doses of $\mathrm{CO}$, we found that among the 16 subjects the increase in DPG was not significant (FIGURE 2).

This latter finding led us to scrutinize the human data more closely. Upon plotting the change in DPG (i.e., change in DPG during CO exposure minus the change seen over the six-hour control period) against the peak $\mathrm{COHb}$ level produced in our subjects, the cause and significance of the difference in response between the two groups of subjects became apparent. After examining those subjects displaying an increase in DPG, it was clear that the peak level of $\mathrm{COHb}$ (FIGURE 3) was considerably lower than COHb levels in the second group of subjects. Apparently the change in DPG levels is inversely related to peak COHb 
concentrations ( $r=-0.48, \mathrm{p},<0.05$ ). This is consistent with our findings in rats; i.e., significant elevations in DPG accompanied only the lower $C O$ dose regimens (TABLE 1 ).

\section{Discussion}

It is now known that reduced (deoxy) hemoglobin has a much higher binding affinity for DPG than either oxy- or carboxyhemoglobin. ${ }^{14}$ The latter two forms of hemoglobin have approximately equal affinities for DPG. The increased affinity of deoxyhemoglobin for DPG has suggested one mechanism for regulation of DPG levels. ${ }^{8,15}$ In normal venous blood about $25-30 \%$ of the hemoglobin is in the reduced form. Much of the DPG may be bound to reduced hemoglobin in the venous blood, while most of the remainder of the DPG is free within the cell (the column on the right in (FIGURE 4) is total DPG and indicates what is assayed). In common hypoxias we find an increased proportion of reduced versus oxygenated hemoglobin (the darkened portions of bar graphs in FIGURE 4). With this increased reduced hemoglobin we have an increase in potential DPGbinding sites and thus an increased amount of DPG bound to reduced hemoglobin. Those mechanisms controlling DPG concentrations in the red cell would presumably respond only to changes in free DPG levels. As more of the free DPG becomes bound, there is a drop in free DPG within the erythrocyte. Thus, DPG synthesis may be activated so as to maintain free DPG at its original level. Accordingly, the increase in the bound DPG means a net increase in the total DPG. This hemoglobin-binding mechanism is probably a factor in the elevation of DPG in the common hypoxias.

However, we often see increases in DPG in anemias ${ }^{8}$ that are greater than this mechanism alone could account for. In an attempt to locate a possible second mechanism, we have sought to produce a type of hypoxia that does not increase the number of available binding sites. Carboxyhemoglobin has the same low affinity for DPG as oxyhemoglobin. Thus when we load an organism with CO, we produce hypoxia by binding a portion of hemoglobin so that it will no longer transport oxygen; secondly, we also produce the Haldane (leftward) shift of the $\mathrm{O}_{2}$ dissociation curve; thirdly, we also decrease the amount of hemoglobin available for binding DPG (the sum of this potential decrease is represented by the clear areas of the third row of bar graphs in FIGURE 4). Hence, the DPG bound to hemoglobin should be either normal or decreased. The amount of free (unbound) DPG should remain unchanged. Therefore, considering only the hemoglobin-binding mechanism, if $\mathrm{CO}$ hypoxia produces any change, it should cause a somewhat reduced amount of total DPG. There would be no way to account for our observed increases in total DPG. On the other hand, if some other type of reaction is triggered by hypoxia to raise the free DPG levels, we might find an increase in the amount of total DPG. The finding that-at least at the lower levels of carboxyhemoglobin-induced hypoxia-we see an increase in DPG suggests a second regulatory device, i.e., hypoxia-driven enhancement of DPG synthesis, which is not dependent upon an increase in reduced hemoglobin. Thus the two mechanisms controlling DPG concentrations are simultaneously acting in different directions. It would appear that, of the two drives governing DPG concentrations, at higher COHb levels the decrease in available binding sites suppresses the DPG increase more potently than the hypoxia mechanism drives DPG synthesis.

It is clear that a third variable, i.e., the $\mathrm{pH}$ of the blood, affects DPG levels. A decrease in $\mathrm{pH}$ results in a decrease in DPG concentrations. However, we do not believe that in the studies presented here that $\mathrm{pH}$ changes are an important 
factor. Astrup's previous work clearly demonstrates that with $\mathrm{CO}$ loadings averaging $15 \%$ ". . . insignificant and nonsystematic changes in blood acid-base occurred." 16 Further, in our studies with rats the animals most likely to have a respiratory alkalosis were those receiving the higher doses of $\mathrm{CO}$, and these were the rats that did not show a significant increase in DPG.

These findings tend to emphasize once more the complex "fine tuning" mechanisms that modulate biological systems. These mechanisms are suggested by the action of the control systems that are operative in carboxyhemoglobin's affect upon DPG. Not only must the hemoglobin binding and hypoxia mechanisms be qualitatively considered, but also their relative influence must be evaluated in any attempt to understand the role of DPG in modulating the erythrocyte's gas transport function.

\section{References}

1. Rapport, S. \& G. M. Guest. 1941. J. Biol. Chem. 138: 269.

2. Chanutin, A. \& R. R. Curnish. 1967. Arch. Biochem. Biophys. 121: 96.

3. Benesch, R., R. E. Benesch \& C. I. Pu. 1968. Proc. Nat. Acad. Sci. 59: 526.

4. MARSE, M., D. E. Cassels \& M. Holder. 1950. J. Clin. Invest. 29: 1098.

5. Dill, D. B., A. V. Bock, C. Canbert, A. Folling, L. M. Hurxthal \& L. J. Henderson. 1928. J. Biol. Chem. 78: 191.

6. Kennedy, A. C. \& D. J. Valtis. 1954. J. Clin Invest. 33: 1372.

7. Hurtado, A. 1964. In Handbook of Physiology. Sect. 4. Adaptation to the Environment. D. B. Dill, Ed. American Physiological Society, Washington, D. C.

8. Eaton, J. W., G. J. Brewer, J. S. Schultz \& C. F. Sing. 1970. In Red Cell Metabolism and Function. G. J. Brewer, Ed. Plenum Press. New York.

9. Jones, R. H., M. F. Ellicott, J. B. Cadigan \& E. A. Gaensler. 1958. J. Lab. Clin. Med. $51(4)$ : 553.

10. Collison, H. A., F. L. Rodkey \& J. D. O'Neal. 1968. Clin. Chem. 14(2): 162.

11. BARTLETT, G. R. 1959. J. Biol. Chem. 234: 469.

12. Eaton, J. W., G. J. Brewer \& R. F. Grover. 1969. J. Lab. Clin. Med. 73: 603.

13. Brewer, G. T., J. W. Eaton \& B. D. Dinman. 1969. J. Lab. Clin. Med. (Abstr.)

14. Chanutin, A. Fösvars Med. 5: 159.

15. Eaton, G. J. \& G. J. Brewer. 1968. Proc. Nat. Acad. Sci. $61: 756$.

16. Mulhausen, R., P. Astrup \& K. Mellemgand, 1968. Scandinav. J. Lab. Clin. Invest. Suppl. 103. 22: 9-15. 